\title{
L0 Norm Based Sparse Regularization for Non-invasive Infarct Detection using ECG Signal
}

\author{
Sandesh Ghimire, Linwei Wang \\ Computational Biomedicine Lab, Rochester Institute of Technology, NY, USA
}

\begin{abstract}
Non-invasive reconstruction of infarcts inside the heart from ECG signals is an important and difficult problem due to the need to solve a severely ill-posed inverse problem. To overcome this ill-posedness, various sparse regularization techniques have been proposed and evaluated for detecting epicardial and transmural infarcts. However, the performance of sparse methods in detecting non-transmural, especially endocardial infarcts, is not fully explored. In this paper, we first show that the detection of non-transmural endocardial infarcts poses severe difficulty to the prevalent algorithms. Subsequently, we propose a novel sparse regularization technique based on a variational approximation of LO norm. In a set of simulation experiments considering transmural and endocardial infarcts, we compare the presented method with total variation minimization and L1 norm based regularization techniques. Experiment results demonstrated that the presented method outperformed prevalent algorithms by a large margin, particularly when infarction is entirely on the endocardium.
\end{abstract}

\section{Introduction}

Inverse electrocardiography (ECG) refers to the noninvasive reconstruction of electrical activity inside the heart from ECG signals. It has been shown that inverse ECG can be used to detect or quantify myocardial infarcts $[1,2]$. The main challenge in inverse ECG is the need to solve a severely ill-posed problem. To address this problem, various regularization techniques have been used in the literature. For the purpose of infarct detection, sparse regularization in the spatial gradient domain of the action potential has been shown to be effective [2]. It is based on the idea that between depolarization and repolarization (i.e. ST segment of ECG), the gradient of action potential is expected to be close to zero everywhere except along the border of an infarct in between viable active tissue and necrotic inactive tissue. L1 norm penalty was used to enforce sparsity to the gradient of action potential and epicardial potential was obtained as inverse solution in $[3,4]$. In $[1,2]$, total-variation minimization was used to enforce sparsity and the inverse solution obtained was applied to quantify transmural infarcts at different locations of the heart. However, the performance of sparse regularization based inverse ECG methods in the presence of nontransmural infarcts, especially endocardial infarcts, remains largely unexplored.

In this paper, we first examine the performance of prevalent sparse inverse ECG methods in detecting transmural versus endocardial infarcts and show that they perform poorly in the endocardial case. We then present a novel L0-norm based sparse regularization method and compare its performance against prevalent algorithms regarding detection of infarcts in both transmural and endocardial cases. L0-norm based sparse regularization is realized in a Bayesian setting where a sparse prior based on a generalized normal distribution is used. To obtain a closed form for the posterior distribution, we derive a variational lower bound of the generalized normal distribution and solve for the posterior distribution using this lower bound by the Expectation Maximization (EM) method.

Simulation experiments were conducted for the detection of transmural and endocardial infarcts of various locations, where we evaluated and compared the presented L0norm based regularization (LOR) with L1-norm based regularization (L1R) and total-variation minimization (TVM). Experiment results show that the presented algorithm, LOR, outperforms L1R and TVM in identifying both transmural and endocardial infarcts, but while the improvement of accuracy is significantly higher in endocardial cases.

\section{Methods}

Transmural action potential is used as the cardiac source model (unknown variable) in this study. The relation between the ECG data and the transmural action potential can be described by a quasi-static approximation of the Maxwell's equations for an electromagnetic field $[5,6]$. Solving these equations numerically on subject specific heart-torso models, a linear forward model can be obtained as

$$
\mathbf{y}_{k}=\mathbf{H} \mathbf{u}_{k}
$$


where $\mathbf{H}$ is called the forward matrix and $\mathbf{y}_{k}$ and $\mathbf{u}_{k}$ denote a vector of ECG measurements and potential inside heart respectively at any time instant $k$. The inverse problem involves estimation of $\mathbf{u}_{k}$ given measurements $\mathbf{y}_{k}$ for all time $k$. In this paper, we solve this separately for individual times instant $k$. For clarity, the subscript $k$ is dropped from the formulations in the remainder of the paper.

\subsection{Bayesian formulation of the inverse ECG problem}

In a Bayesian formulation, we are interested in obtaining the posterior distribution of the action potential given the ECG data which, according to the Baye's theorem, is formulated as:

$$
p(\mathbf{u} \mid \mathbf{y})=\frac{p(\mathbf{y} \mid \mathbf{u}) p(\mathbf{u})}{p(\mathbf{y})}
$$

In (2), the likelihood function is assumed to be a Gaussian distribution that incorporates the forward model as:

$$
p(\mathbf{y} \mid \mathbf{u}, \beta)=\mathcal{N}\left(\mathbf{y} \mid \mathbf{H u}, \beta^{-1} \mathbf{I}\right)
$$

where $\beta^{-1}$ is the variance of an additive Gaussian noise. We let the precision (inverse variance) $\beta$ to be an unknown parameter following a Gamma distribution.

As explained earlier, the prior distribution of $\mathbf{u}$ is designed to impose sparsity in its gradient domain. Let us define a vector $\mathbf{x}=\mathbf{D u}$, to denote the gradient of action potential with matrix $\mathbf{D}$ being the gradient operator on the 3D cardiac mesh. To mimic the L0-norm constraint on $\mathbf{x}$ without having to solve NP hard problem, we propose a generalized Gaussian distribution for each component of $\mathbf{x}$. Let $\mathbf{x}=\left(x_{1}, x_{2}, \ldots x_{N}\right)$ be a vector with independent components each following a generalized normal distribution with the same parameters $\alpha, p$. We have,

$$
p(\mathbf{x} \mid \alpha)=\prod_{i} \frac{C}{\alpha} \exp \left(\frac{-\left|x_{i}\right|^{p}}{\alpha^{p}}\right)
$$

where $p \sim 0$ approximates a L0 norm constraint in a probabilistic setting.

\subsection{Variational approximation}

Using a prior distribution as defined in eq.(4), the posterior distribution of $\mathbf{u}$ as defined in (2) is analytically intractable. Hence, we propose a variational approximation of the original prior distribution.

\section{Theorem 1}

If the pdf of a random vector $\mathbf{x}$ is given by eq.(4), then,

$$
p(\mathbf{x} \mid \alpha) \geq \frac{C^{N}}{\alpha^{N}} \exp \left(-\frac{\mathbf{x}^{T} \mathbf{A x}}{2}\right) \exp \left(-\frac{2-p}{2}\left(\frac{\alpha^{2}}{p}\right)^{\frac{p}{p-2}} \sum_{i} \lambda_{i}^{\frac{p}{p-2}}\right)
$$

The proof of Theorem 1 makes use of the Legendre transform and convex analysis, but has been omitted due to page constraints. Theorem 1 provides a lower bound for $p(\mathbf{x} \mid \alpha)$, which is then used as the prior distribution of $\mathbf{x}$. By replacing $\mathbf{x}=\mathbf{D u}$ in the right hand side of eq. (5), the prior of $\mathbf{u}$ is given by

$$
p(\mathbf{u} \mid \boldsymbol{\lambda}, \alpha)=\frac{C^{N}}{\alpha^{N}} \exp \left(-\frac{\mathbf{u}^{T} \mathbf{D}^{T} \mathbf{A D u} \mathbf{u}}{2}\right) \exp \left(-\frac{2-p}{2}\left(\frac{\alpha^{2}}{p}\right)^{\frac{p}{p-2}} \sum_{i} \lambda_{i}^{\frac{p}{p-2}}\right)
$$

\subsection{Inference}

We note that both the prior and likelihood distributions have parameters $\alpha, \beta, \boldsymbol{\lambda}$ for which we do not have reliable knowledge. To estimate the posterior distribution over $\mathbf{u}$, therefore, we need to estimate these unknown parameters. This is done by the Expectation Maximization (EM) procedure [7] that consists of the following iterations:

a. E-step: In this step, values of parameters are used from the previous step. If we denote previous parameter values as $\alpha_{\text {old }}, \beta_{\text {old }}, \boldsymbol{\alpha}_{\text {old }}$, then we calculate the posterior distribution with those fixed parameters $q(\mathbf{u})=$ $p\left(\mathbf{u} \mid \mathbf{y}, \alpha_{\text {old }}, \beta_{\text {old }}, \boldsymbol{\lambda}_{\text {old }}\right)$. This gives us a function $L$ as:

$$
L(\alpha, \beta, \boldsymbol{\lambda})=E_{q(\mathbf{u})}[\log (p(\mathbf{u}, \mathbf{y} \mid \alpha, \boldsymbol{\lambda}, \beta))+\log (\beta)]
$$

where $E_{q(\mathbf{u})}[]$ denotes the expectation with respect to distribution $q(\mathbf{u})$.

b. M-step: This step consists of the following maximization

$$
\alpha_{\text {new }}, \beta_{\text {new }}, \boldsymbol{\lambda}_{\text {new }}=\underset{\alpha, \beta, \boldsymbol{\lambda}}{\operatorname{argmax}} L(\alpha, \beta, \boldsymbol{\lambda})
$$

These two steps are repeated until convergence. At convergence, we obtain both the estimate of parameters $\hat{\alpha}, \hat{\beta}, \hat{\lambda}$ and the posterior distribution $p(\mathbf{u} \mid \mathbf{y}, \hat{\boldsymbol{\alpha}}, \hat{\boldsymbol{\beta}}, \hat{\boldsymbol{\alpha}})$.

From the mean of the estimated posterior distribution of $\mathbf{u}$, regions with the value of action potential below a threshold are identified as infarcts.

\section{Experiments and Results}

Experiments were performed on MRI derived hearttorso model with infarcts set according to the AHA 17 segment model on the left ventricle. ECG signals were simulated form the forward model and corrupted with 20 $\mathrm{dB}$ Gaussian noise for inverse reconstruction. On transmural and endocardial infarcts, we compared the performance of the presented L0 norm based regularization (LOR) with existing L1 norm based regularization (L1R) and total variation minimization (TVM) methods. Metrics used to compare performance are defined as follows:

1) True Positive (Hit): If the reconstruction from the algorithm contains (fully or partially) the actual region of the infarct, it is considered a hit. Out of all tested cases, number of hits is calculated for each method. 

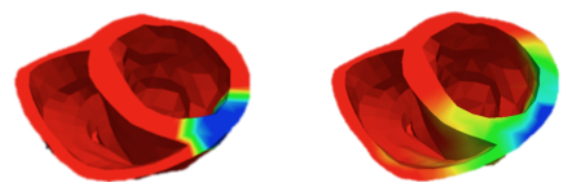

Seg-1

Ground Truth

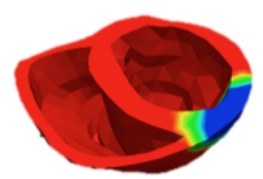

LO Reg.

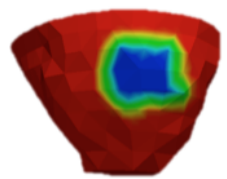

Ground Truth

Seg-12

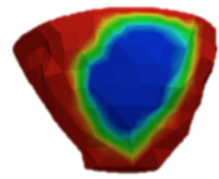

LO Reg.

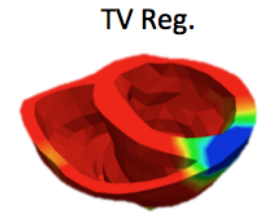

L1 Reg $\max$

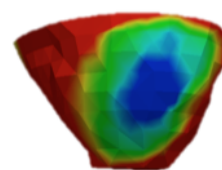
TV Reg.

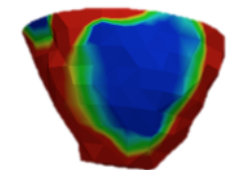

L1 Reg.
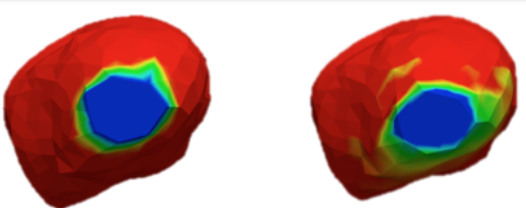

Seg-17

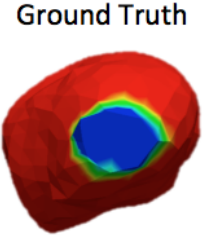

LO Reg.
TV Reg.

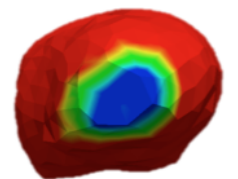

L1 Reg.

Figure 1. Reconstruction of transmural infarcts using total variation, L0 norm and L1 norm based sparse regularization in gradient domain.

2) False positive patch per hit (FP): If the reconstruction contains false positive patches in addition to the actual region of infarct, the number of false positive patches per hit is calculated. For example, in Fig. 2, Seg-12, the reconstruction by using L1R contains actual infarct region but has two more false positive patches.

3) Dice coefficient $(\mathrm{DC})=2(T \cap R) /(T \cup R)$, where $T$ and $R$ denote, respectively, true and reconstructed regions of infarct.

Fig. 1 compares the reconstruction of transmural infarcts by the proposed LOR with that by L1R and TVM in three segments: Segment 1, 12 and 17. It should be noted that

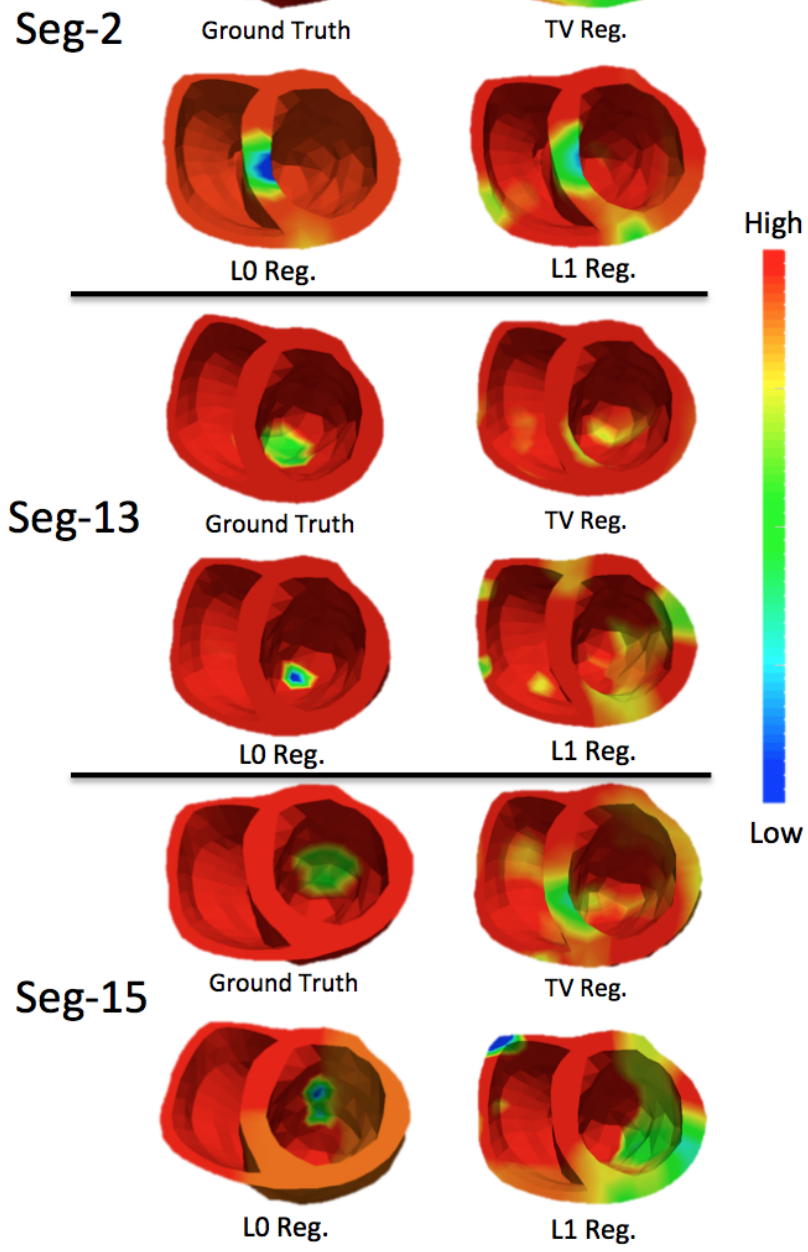

Figure 2. Reconstruction of infarcts that lie entirely on the endocardium using total variation, L0 norm and L1 norm based sparse regularization.

all three methods performed well although the reconstruction by L0 norm seems slightly closer to the ground truth. Similar a conclusion can be drawn from the hit rate in the top half of Table 1. Interestingly, TVM appeared to suffer from a higher presence of false positives, as evidenced by the significantly higher number of false positives (doubling those of the other two methods) and the significantly reduced dice coefficient (nearly half of those of the other two methods).

Fig.2 shows some examples comparing the three algorithms when the infarct lies entirely on the endocardium. In all three segments, LOR reconstructed scar at a location consistent with the ground truth and without false posi- 
Table 1. Performance of three sparse algorithms for infarct detection $(\mathrm{Hit}=$ True Positive, $\mathrm{FP}=$ False positive patches per hit, $\mathrm{DC}=$ Dice coefficient)

\begin{tabular}{|l|c||c|c|r|}
\hline \multicolumn{2}{|c|}{ Cases $\backslash$ Methods } & TVM & L1R & L0R \\
\hline \hline \multirow{4}{*}{$\begin{array}{l}\text { Transmural } \\
\text { infarct }\end{array}$} & Hit & $\begin{array}{c}15 / 17 \\
(88.2 \%)\end{array}$ & $\begin{array}{c}15 / 17 \\
(88.2 \%)\end{array}$ & $\begin{array}{r}\mathbf{1 6} / \mathbf{1 7} \\
(\mathbf{9 4 . 1} \%)\end{array}$ \\
\cline { 2 - 5 } & FP & 0.6 & 0.33 & $\mathbf{0 . 3 1}$ \\
\cline { 2 - 5 } & DC & 0.39 & 0.8 & $\mathbf{0 . 9 5}$ \\
\hline \multirow{4}{*}{$\begin{array}{l}\text { Endocardial } \\
\text { infarct }\end{array}$} & Hit & $6 / 17$ & $6 / 17$ & $\mathbf{1 1 / 1 7}$ \\
\cline { 2 - 5 } & & $(35.3 \%)$ & $(35.3 \%)$ & $\mathbf{( 6 4 . 7 \% )}$ \\
\cline { 2 - 5 } & FP & 2.67 & 2.33 & $\mathbf{0 . 4 5}$ \\
\hline \multicolumn{2}{|c|}{} & 0.158 & 0.17 & $\mathbf{0 . 2 9}$ \\
\hline
\end{tabular}

tives. When infarct was in segment 2, both TVM and L1R did detect the infarct at the correct location, but with a lot of false positives. When infarct was in segment 13, L1R failed completely while TVM reconstructed the infarct but with false positives. In Segment 15, neither TVM nor L1R could detect the infarct at the correct location. Similar observations can be made on the bottom half of Table 1 . The hit rate and dice coefficient of LOR is almost twice and false positives are five times smaller compared to the other two algorithms when the infarct is completely endocardial. Unlike in transmural case, both L1R and TVM showed very limited accuracy in detecting endocardial infarcts.

In summary, for both transmural and endocardial cases, the presented LOR norm shows improved accuracy than TVM and L1R. This improvement is significant for nontransmural endocardial infarcts. It is noteworthy that six out of 17 segments could not be reconstructed by any of the three methods. These segments were in the septum and the lateral side of the left ventricle. We speculate that this might be because the contribution to the ECG is small from sources at these locations of the heart. Since sparse algorithms work by pruning out the less important sources, it might be possible that these sources were pruned out even though the actual sources lie in those locations. However, thorough analysis is needed to reach any conclusion.

\section{Conclusions}

This paper developed a Bayesian approach using an approximation of a LO norm based prior distribution and compared its performance to two prevalent sparse methods in detecting transmural and non-transmural endocardial infarcts. Experiments showed that the performance of L0 norm based regularization outperformed the other two sparse regularization techniques in detecting both the transmural and endocardial infarcts, although the improvement in performance is much more significant in the endocardial case where the other two algorithms performed poorly. Future works will examine this observation in a larger number of experiments, especially using real data.
Regarding locations at which none of the sparse algorithms under study succeeded at reconstruction, we anticipate that better approximation of LO norm and consequently better sparse regularization might be possible. However, this observation also raises the question if sparse methods alone are sufficient for inverse ECG because LO is the sparsest one could possibly go. Lastly, our results showed that the effectiveness of a sparse algorithm depends on both the location and transmurality of the infarct, both of which are reflected in the behavior of forward matrix. Therefore, it would be worthwhile to understand the precise connection (preferably a rigorous mathematical relation) between the success of the reconstruction and the location as well as the transmurality of the infarct so as to understand algorithms along with the conditions under which they perform well.

\section{Acknowledgements}

This work is supported in part by the National Institutes of Health [grant no: R21HL125998] and the National Science Foundation [grant no: ACI-1350374].

\section{References}

[1] Xu J, Sapp JL, Dehaghani AR, Gao F, Wang L. Variational bayesian electrophysiological imaging of myocardial infarction. In International Conference on MICCAI. Springer, 2014; 529-537.

[2] Xu J, Dehaghani AR, Gao F, Wang L. Noninvasive transmural electrophysiological imaging based on minimization of total-variation functional. IEEE transactions on medical imaging 2014;33(9):1860-1874.

[3] Ghosh S, Rudy Y. Application of 11-norm regularization to epicardial potential solution of the inverse electrocardiography problem. Annals of biomedical engineering 2009; 37(5):902-912.

[4] Shou G, Xia L, Liu F, Jiang M, Crozier S. On epicardial potential reconstruction using regularization schemes with the 11-norm data term. Physics in medicine and biology 2010; 56(1):57.

[5] Wang L, Zhang H, Wong KC, Liu H, Shi P. Physiologicalmodel-constrained noninvasive reconstruction of volumetric myocardial transmembrane potentials. IEEE Transactions on Biomedical Engineering 2010;57(2):296-315.

[6] Wang L, Dawoud F, Yeung SK, Shi P, Wong KL, Liu H, Lardo AC. Transmural imaging of ventricular action potentials and post-infarction scars in swine hearts. IEEE TMI 2013;32(4):731-747.

[7] Dempster AP, Laird NM, Rubin DB. Maximum likelihood from incomplete data via the em algorithm. Journal of the royal statistical society Series B methodological 1977;1-38.

Address for correspondence:

Sandesh Ghimire-sg9872@rit.edu

Rochester Institute of Technology

1 Lomb Memorial Drive, Rochester, NY-14623, USA 